Modern Physics Letters B

Vol. 27 No. 23 (2013) 1392001 (1 page)

(C) World Scientific Publishing Company

DOI: $10.1142 /$ S0217984913920012

\title{
ERRATA
}

\section{THEORETICAL STUDY OF TRANSMISSION SPECTRUM IN CAVITY-WAVEGUIDE SIDE-COUPLED SYSTEMS}

\author{
[Mod. Phys. Lett. B, Vol. 27, No. 22 (2013) 1350157 (12 pages)] \\ CHUANBO DONG, TAO WANG, WEI YAN and XIAOMING LI
}

We would like to make the following corrections to this article.

(1) The corresponding author of the article should be revised as TAO WANG, and the email should be revised as: wangtao@hust.edu.cn. The main work of this article is CHUANBO DONG. However, the supervisor is TAO WANG. The reason we use CHUANBO DONG as corresponding author initially is for instant communication and convenient contact.

(2) Line 1, page 1350157-2, "plasmonic fields" should be revised as "plasmonic effects."

(3) Page 1350157-6, Eq. (5c) does not coincide with the equation number in our manuscript. Equation "(5c)" should be revised as Eq. "(6)." Otherwise, it would cause confusion to the readers.

$$
\begin{gathered}
r=\frac{s_{-1, \text { total }}}{s_{+1}}=r_{1}+\frac{t_{1} r_{2} t_{1} e^{-j \cdot 2 \beta D}}{1-r_{1} r_{2} e^{-j \cdot 2 \beta D}}, \\
t=\frac{s_{-4, \text { total }}}{s_{+1}}=\frac{t_{2} t_{1} e^{-j \beta D}}{1-r_{1} r_{2} e^{-j \cdot 2 \beta D}}=\frac{\left|t_{1}\right|\left|t_{2}\right| e^{-j \cdot \beta(2 L+D)}}{1-\left|r_{1}\right|\left|r_{2}\right| e^{-j \cdot 2 \beta(L+D)}}, \\
T=|t|^{2}=\frac{\left|t_{2} t_{1}\right|^{2}}{|1-| r_{1}|\cdot| r_{2}\left|e^{-j \cdot 2 \varphi}\right|^{2}} .
\end{gathered}
$$

(4) Similar to error (3), page 1350157-8, Eq. "(6)" should be revised as Eq. "(6')."

$$
T^{\prime}=\left|\frac{s_{-4}}{s_{+1}}\right|^{2}=\left|t_{2}^{\prime} t_{1}^{\prime}\right|^{2} .
$$

We thank the authors for directing our attention to the above corrections. 